\title{
A NEW PROOF OF THE CONSTRUCTION THEOREM FOR STONE ALGEBRAS
}

\author{
TIBOR KATRIŇÁK
}

\begin{abstract}
A simple proof is given of Chen's and Grätzer's theorem, which gives a method to construct a Stone algebra from a Boolean algebra and a distributive lattice with 1 by certain connective conditions between the two given lattices.
\end{abstract}

C. C. Chen and G. Grätzer [1] proved originally the Construction Theorem for Stone algebras. In [3] we proved by different method the Construction Theorem for a larger class of structures with pseudocomplementation than the class of Stone algebras. Modifying the method from [1] we have proved in [4] the Construction Theorem for distributive lattices with pseudocomplementation. The proofs of all mentioned theorems are rather complicated. G. Grätzer in his book [2] set a task (Problem 55): "Find a direct (less-computational) proof of the Construction Theorem for Stone algebras".

In this note we shall give an answer to this problem. It will be a simpler proof of the Construction Theorem.

Preliminaries. A universal algebra $\left\langle L ; \cup, \cap,{ }^{*}, 0,1\right\rangle$ of type $\langle 2,2,1$, $0,0\rangle$ is called a distributive p-algebra iff $\langle L ; \cup, \cap, 0,1\rangle$ is a bounded distributive lattice such that for every $a \in L$ the element $a^{*}$ is the pseudocomplement of $a$, i.e. $x \leqq a^{*}$ iff $a \cap x=0$. A distributive $p$-algebra satisfying the Stone identity $x^{*} \cup x^{* *}=1$ is called a Stone algebra. The standard results on Stone algebras may be found in [2].

For a Stone algebra $L$ define the set $B(L)=\left\{x \in L: x=x^{* *}\right\}$ of closed elements. The partial ordering of $L$ partially orders $B(L)$ and turns the latter into a Boolean algebra $\langle B(L) ; \cup, \cap, *, 0,1\rangle$. Another significant subset of a Stone algebra $L$ is the set of dense elements $D(L)=\{x \in L$ : $\left.x^{*}=0\right\} . D(L)$ is a filter (dual ideal) in $L$.

Let $F(D)$ denote the set of all filters of $D$ ordered by the set inclusion. $F(D(L))$, for a Stone algebra $L$, is a distributive lattice. Finally define a

Received by the editors July 26, 1972 and, in revised form, November 27, 1972.

AMS (MOS) subject classifications (1970). Primary 06A25, 06A35; Secondary 06A40.

Key words and phrases. Distributive lattice with pseudocomplementation, $p$-algebra, Stone algebra, Stone lattice, Boolean algebra, pseudocomplement, dense element, closed element.

(c) American Mathematical Society 1973 
mapping $\varphi(L): B(L) \rightarrow F(D(L))$ by

$$
\varphi(L): a \rightarrow\left\{x \in D(L): x \geqq a^{*}\right\} .
$$

A $\{0,1\}$-homomorphism (of a bounded lattice into another one) is a homomorphism taking zero into zero and unit into unit.

The relationship between $B(L), D(L)$ and $\varphi(L)$ is expressed in the following.

Theorem ([1], [2]). Let $L$ be a Stone algebra. Then $B(L)$ is a Boolean algebra, $D(L)$ is a distributive lattice with 1 , and $\varphi(L)$ is a $\{0,1\}$-homomorphism of $B(L)$ into $F(D(L))$. The triple $\langle B(L), D(L), \varphi(L)\rangle$ characterizes $L$ up to isomorphism.

The construction. Now we can give a new and simpler proof of the following.

CONSTRUCtion Theorem ([1], [2]). Given a Boolean algebra $\langle\boldsymbol{B}$; $\left.\cup, \cap,{ }^{\prime}, 0,1\right\rangle$, a distributive lattice $D$ with 1 , and $a\{0,1\}$-homomorphism $\varphi: B \rightarrow F(D)$, there exists a Stone algebra $L$ whose triple is $\langle B, D, \varphi\rangle$.

Proof. Let $[t]=\{x \in D: t \in D$ and $x \geqq t\}$ for each $t \in D$. Put $a \theta=a^{\prime} \varphi$ for $a \in B$. Then $0 \theta=D, 1 \theta=[1),(a \cap b) \theta=a \theta \cup b \theta$ and $(a \cup b) \theta=a \theta \cap b \theta$. Let $\left\langle F_{d}(D) ; \vee, \wedge\right\rangle$ denote the dual lattice to the distributive lattice $\langle F(D) ; \cup, \cap\rangle$ of all filters of $D$, i.e. $J \vee K=J \cap K, J \wedge K=J \cup K$ for $J$, $K \in F(D)$.

Since $a \varphi(a \in B)$ belongs to the centre of $F(D)$, for every $x \in D$, there exists $t \in D$ such that $[t]=a \varphi \cap[x)$. Denote the element $t$ by $x \rho_{a}$.

Set

$$
L=\{\langle a, a \theta \cup[x)\rangle: a \in B, x \in D\} .
$$

$L$ is a subset of the direct product $B \times F_{d}(D)$. We show first that $L$ is a sublattice of $B \times F_{d}(D)$. Let $\langle a, a \theta \cup[x)\rangle,\langle b, b \theta \cup[y)\rangle \in L$. Then we have in the lattice $B \times F_{d}(D)$

$$
\begin{aligned}
& \langle a, a \theta \cup[x)\rangle \cap\langle b, b \theta \cup[y)\rangle \\
& \quad=\langle a \cap b, a \theta \cup b \theta \cup[x) \cup[y)\rangle=\langle a \cap b,(a \cap b) \theta \cup[x \cap y)\rangle
\end{aligned}
$$

and

(3) $\langle a, a \theta \cup[x)\rangle \cup\langle b, b \theta \cup[y)\rangle=\langle a \cup b,(a \theta \cup[x)) \cap(b \theta \cup[y))\rangle$.

By distributivity of $F(D)$ we obtain

$$
\begin{aligned}
(a \theta \cup[x)) & \cap(b \theta \cup[y)) \\
& =(a \theta \cap b \theta) \cup(a \theta \cap[y)) \cup([x) \cap b \theta) \cup([x) \cap[y)) \\
& =(a \cup b) \theta \cup\left[y \rho_{a^{\prime}} \cap x \rho_{b^{\prime}} \cap(x \cup y)\right) .
\end{aligned}
$$


(2)-(4) implies that $L$ is a sublattice of $B \times F_{d}(D)$. Hence $L$ is distributive. It is easy to see that

(5) $\langle a, a \theta \cup[x)\rangle \leqq\langle b, b \theta \cup[y)\rangle \quad$ iff $a \leqq b$ and $a \theta \cup[x] \supseteq b \theta \cup[y)$ in $L . L$ is bounded and

$$
\langle 0, D\rangle \leqq\langle a, a \theta \cup[x)\rangle \leqq\langle 1,[1)\rangle
$$

holds.

$L$ is pseudocomplemented and

$$
\langle a, a \theta \cup[x)\rangle^{*}=\left\langle a^{\prime}, a^{\prime} \theta\right\rangle
$$

is true. Really,

$$
\langle a, a \theta \cup[x)\rangle \cap\left\langle a^{\prime}, a^{\prime} \theta\right\rangle=\left\langle 0, a \theta \cup a^{\prime} \theta \cup[x)\right\rangle=\langle 0, D\rangle .
$$

If $\langle a, a \theta \cup[x)\rangle \cap\langle b, b \theta \cup[y)\rangle=\langle 0, D\rangle$ then $b \leqq a^{\prime}$. The latter implies $b \theta \supseteq a^{\prime} \theta$. Therefore, by (5),

$$
\langle b, b \theta \cup[y)\rangle \leqq\left\langle a^{\prime}, a^{\prime} \theta\right\rangle .
$$

Thus (7) is proved.

Now it is easy to show that $L$ is a Stone algebra.

(7) implies

$$
\begin{aligned}
& B(L)=\{\langle a, a \theta\rangle ; a \in B\} \text { and } \\
& D(L)=\{\langle 1,[x)\rangle ; x \in D\} .
\end{aligned}
$$

Identifying $a \in B$ with $\langle a, a \theta\rangle$ and $d \in D$ with $\langle 1,[d)\rangle$ we can verify $B(L)=B$ and $D(L)=D$. Finally we prove $\varphi(L)=\varphi$. By (5),

$$
\langle 1,[x)\rangle \geqq\left\langle a^{\prime}, a^{\prime} \theta\right\rangle \quad \text { iff }[x) \subseteq a^{\prime} \theta=a \varphi .
$$

Hence $x \in D$ and $x \geqq a^{*}$ in $L$ iff $x \in a \varphi$. Thus $\varphi(L)=\varphi$ and the theorem is proved.

REMARK 1. Another simplification can be obtained if $D$ is supposed to be bounded. Let $s$ be the smallest element of $D$. It is known that $a \varphi$ $(a \in B)$ is a principal filter of $D$, i.e. there exists $t \in D$ such that $a \varphi=$ $[t)$. Put $\left[n_{a}\right)=a \theta$. Then $L$ can be defined in the following way.

$$
L=\left\{\langle a, d\rangle ; s \leqq d \leqq n_{a}\right\} .
$$

$L$ is a sublattice of the direct product $B \times D$ and the relations (2), (3), 
(5)-(7) are changed into

$$
\begin{aligned}
\langle a, d\rangle \cap\langle b, e\rangle & =\langle a \cap b, d \cap e\rangle ; \\
\langle a, d\rangle \cup\langle b, e\rangle & =\langle a \cup b, d \cup e\rangle ; \\
\langle a, d\rangle & \leqq\langle b, e\rangle \text { iff } a \leqq b \text { and } d \leqq e ; \\
\langle 0, s\rangle & \leqq\langle a, d\rangle \leqq\langle 1,1\rangle ; \\
\langle a, d\rangle^{*} & =\left\langle a^{\prime}, n_{a^{\prime}}\right\rangle
\end{aligned}
$$

REMARK 2. We can compare our construction with that given in [1]. More precisely, let $\langle B, D, \varphi\rangle$ be a triple described in the Construction Theorem. Let $L$ denote the Stone algebra constructed to $\langle B, D, \varphi\rangle$ while $L_{1}$ denotes the Stone algebra corresponding to the same triple in [1]. We recall that

and

$$
L_{1}=\{\langle x, a\rangle ; a \in B, x \in a \varphi\}
$$

$$
\langle x, a\rangle \leqq\langle y, b\rangle \quad \text { iff } a \leqq b, x \leqq y \rho_{a} .
$$

Since $(a \theta \cup[x)) \cap a \varphi=\left[x \rho_{a}\right)$, an easy calculation shows that the mapping $\langle a, a \theta \cup[x)\rangle \rightarrow\left\langle x \rho_{a}, a\right\rangle$ establishes an isomorphism of $L$ onto $L_{1}$.

\section{REFERENCES}

1. C. C. Chen and G. Grätzer, Stone lattices. I: Construction theorems, Canad. J. Math. 21 (1969), 884-894. MR 39 \#4065a.

2. G. Grätzer, Lattice theory. First concepts and distributive lattices, Freeman, San Francisco, Calif., 1971.

3. T. Katriňák, Die Kennzeichnung der distributiven pseudokomplementären Halbverbände, J. Reine Angew. Math. 241 (1970), 160-179. MR 41 \#5253.

4. - Über eine Konstruktion der distributiven pseudokomplementären Verbände, Math. Nachr. 53 (1972), 85-99.

Pavilon Matematiky, Univerztta Komenského, Bratislava 16, Czechoslovakia 